\title{
Sir Andrew J. Wiles Awarded Abel Prize
}

Elaine Kehoe with The Norwegian Academy of
Science and Letters official Press Release
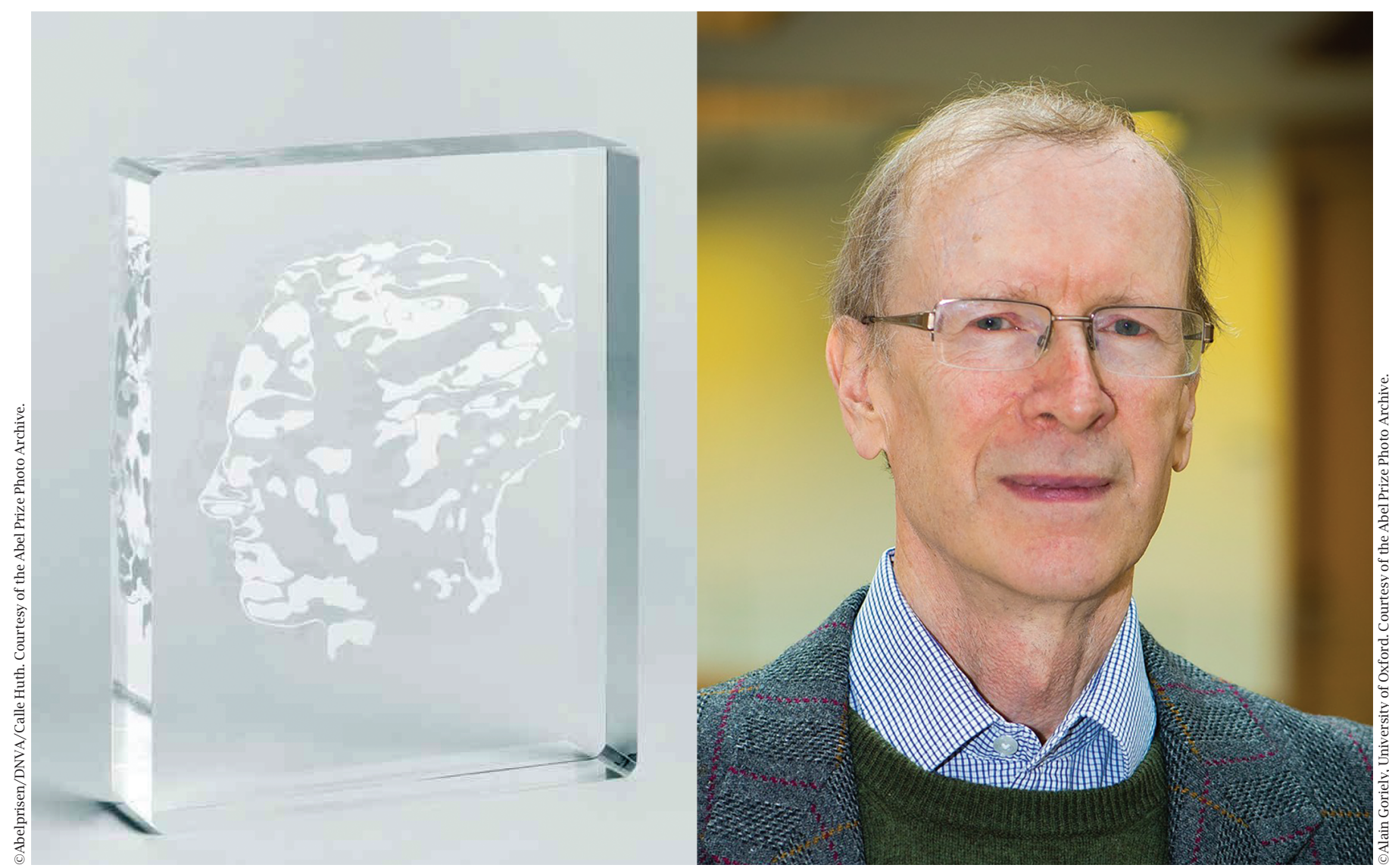

Sir Andrew Wiles received the 2016 Abel Prize at the Oslo award ceremony on May 24.

The Norwegian Academy of Science and Letters has awarded the 2016 Abel Prize to SiR ANDREW J. WILES of the University of Oxford "for his stunning proof of Fermat's Last Theorem by way of the modularity conjecture for semistable elliptic curves, opening a new era in number theory." The Abel Prize is awarded by the Norwegian Academy of Science and Letters. It recognizes contributions of extraordinary depth and influence to the mathematical sciences and has been awarded annually since 2003. It

For permission to reprint this article, please contact:

reprint-permission@ams .org.

DOI: http://dx.doi.org/10.1090/noti1386 carries a cash award of 6,000,000 Norwegian krone (approximately US $\$ 700,000)$.

\section{Citation}

Number theory, an old and beautiful branch of mathematics, is concerned with the study of arithmetic properties of the integers. In its modern form the subject is fundamentally connected to complex analysis, algebraic geometry, and representation theory. Number theoretic results play an important role in our everyday lives through encryption algorithms for communications, financial transactions, and digital security.

Fermat's Last Theorem, first formulated by Pierre de Fermat in the seventeenth century, is the assertion that 
the equation $x^{n}+y^{n}=z^{n}$ has no solutions in positive integers for $n>2$. Fermat proved his claim for $n=4$, Leonhard Euler found a proof for $n=3$, and Sophie Germain proved the first general result that applies to infinitely many prime exponents. Ernst Kummer's study of the problem unveiled several basic notions in algebraic number theory, such as ideal numbers and the subtleties of unique factorization. The complete proof found by Andrew Wiles relies on three further concepts in number theory, namely elliptic curves, modular forms, and Galois representations.

Elliptic curves are defined by cubic equations in two variables. They are the natural domains of definition of the elliptic functions introduced by Niels Henrik Abel. Modular forms are highly symmetric analytic functions defined on the upper half of the complex plane, and naturally factor through shapes known as modular curves. An elliptic curve is said to be modular if it can be parametrized by a map from one of these modular curves. The modularity conjecture, proposed by Goro Shimura, Yutaka Taniyama, and André Weil in the 1950s and 1960s, claims that every elliptic curve defined over the rational numbers is modular.

In 1984, Gerhard Frey associated a semistable elliptic curve to any hypothetical counterexample to Fermat's Last Theorem, and strongly suspected that this elliptic curve would not be modular. Frey's nonmodularity was proven via Jean-Pierre Serre's epsilon conjecture by Kenneth Ribet in 1986. Hence, a proof of the Shimura-Taniyama-Weil modularity conjecture for semistable elliptic curves would also yield a proof of Fermat's

Last Theorem. However, at the time the modularity conjecture was widely believed to be completely inaccessible. It was therefore a stunning advance when Andrew Wiles, in a breakthrough paper published in 1995, introduced his modularity lifting technique and proved the semistable case of the modularity conjecture.

The modularity lifting technique of Wiles concerns the Galois symmetries of the points of finite order in the abelian group structure on an elliptic curve. Building upon Barry Mazur's deformation theory for such Galois representations, Wiles identified a numerical criterion which ensures that modularity for points of order $p$ can be lifted to modularity for points of order any power of $p$, where $p$ is an odd prime. This lifted modularity is then sufficient to prove that the elliptic curve is modular. The numerical criterion was confirmed in the semistable case by using an important companion paper written jointly with Richard Taylor. Theorems of Robert Langlands and Jerrold Tunnell show that in many cases the Galois representation given by the points of order three is modular. By an ingenious switch from one prime to another, Wiles showed that in the remaining cases the Galois representation given by the points of order five is modular. This completed his proof of the modularity conjecture, and thus also of Fermat's Last Theorem.

The new ideas introduced by Wiles were crucial to many subsequent developments, including the proof in 2001 of the general case of the modularity conjecture by Chris- tophe Breuil, Brian Conrad, Fred Diamond, and Richard Taylor. As recently as 2015, Nuno Freitas, Bao V. Le Hung, and Samir Siksek proved the analogous modularity statement over real quadratic number fields. Few results have as rich a mathematical history and as dramatic a proof as Fermat's Last Theorem.

\section{Biographical Sketch}

Andrew J. Wiles was born April 11, 1953, in Cambridge, United Kingdom. He earned his PhD in 1980 at Clare College, Cambridge. He has held positions at Harvard University and Princeton University. In 1985-86, he was a Guggenheim Fellow at the Institut des Hautes Études Scientifiques and at the École Normale Supérieure. From 1988 to 1990 he was a Royal Society Research Professor at the University of Oxford before returning to Princeton. He rejoined Oxford in 2011 as Royal Society Research Professor.

Andrew Wiles is one of the very few mathematiciansif not the only one-whose proof of a theorem has been international headline news. His proof was not only the high point of his career-and an epochal moment for mathematics - but also the culmination of a remarkable personal journey that began three decades before. In 1963, when he was a ten-year-old boy growing up in Cambridge, Wiles found a copy of a book on Fermat's Last Theorem in his local library. He became captivated by the problem-that there are no whole number solutions to the equation $x^{n}+y^{n}=\mathrm{z}^{n}$ when $n$ is greater than 2 -which was easy to understand but which had remained unsolved for three hundred years. "I knew from that moment that I would never let it go," he said. "I had to solve it."

Wiles studied mathematics at Merton College, Oxford, and returned to Cambridge, at Clare College, for postgraduate studies. His research area was number theory, the mathematical field that investigates the properties of numbers. Under the guidance of his advisor, John Coates, he studied elliptic curves, a type of equation that was first studied in connection with measuring the lengths of planetary orbits. Together they made the first progress on one of the field's fundamental conjectures, the Birch and Swinnerton-Dyer conjecture, proving it for certain special cases. Wiles was awarded his $\mathrm{PhD}$ in 1980 for the thesis "Reciprocity laws and the conjecture of Birch and Swinnerton-Dyer".

Between 1977 and 1980 he was an assistant professor at Harvard University, where he started to study modular forms, a separate field from elliptic curves. There he began a collaboration with Barry Mazur, which resulted in their 1984 proof of the main conjecture of Iwasawa theory, a field within number theory. In 1982 he was made a professor at Princeton University.

During the early years of his academic career he was not actively trying to solve Fermat's Last Theorem, nor was anyone else, since the problem was generally regarded as too difficult and possibly unsolvable. A turning point came in 1986 when it was shown that the three-centuriesold problem could be rephrased using the mathematics 
of elliptic curves and modular forms. It was an amazing twist of fate that two subjects that Wiles had specialized in turned out to be exactly the areas that were needed to tackle Fermat's Last Theorem with modern tools. He decided that he would return to the problem that so excited him as a child. "The challenge proved irresistible," he said.

Wiles made the unusual choice to work on Fermat alone rather than collaborating with colleagues. Since the problem was so famous, he was worried that news he was working on it would attract too much attention and he would lose focus. The only person he confided in was his wife, Nada, whom he married shortly after embarking on the proof.

After seven years of intense and secret study, Wiles believed he had a proof. He decided to go public during a lecture series at a seminar in Cambridge, England. He did not announce it beforehand. The title of his talk, "Modular Forms, Elliptic Curves and Galois Representations", gave nothing away, although rumor had spread around the mathematical community and two hundred people were packed into the lecture theater to hear him. When he wrote the theorem up as the conclusion to the talk, the room erupted in applause.

Later that year, however, a referee checking the details of his proof found an error in it. It was devastating for Wiles to contemplate the idea that he had not, in fact, solved Fermat's Last Theorem. He set to work trying to

Congratulations from AMS President Robert Bryant
"On behalf of the American Mathematical Society, it
is a great pleasure to congratulate Sir Andrew Wiles,
recipient of the 2016 Abel Prize.
Professor Wiles' astonishing breakthroughs in
number theory, paving the way for establishing the
Modularity Conjecture of Shimura, Taniyama, and Weil,
have not only led to a new era in the theory of elliptic
curves but have solved long-standing famous problems,
such as Fermat's Last Theorem, and inspired some of
the most remarkable advances in mathematics in the
last one hundred years.
Dr. Wiles' fundamental work in number theory has
implications far beyond its deep consequences in pure
mathematics, deepening our understanding of some of
the most fundamental algorithms that underlie commu-
nications in our modern world and providing enormous
benefits to our society and our world."
- Robert Bryant, AMS President

fix the issue, enlisting one of his former students, Richard Taylor, to help him with the task. After a year's work, Wiles found a way to correct the error. "I had this incredible revelation," a tearful Wiles told a BBC documentary. "It was the most important moment of my working life."

Not only is it rare to announce the proof of a famous theorem, but it is also extremely unusual to go back and fix an error like this because of the mental exhaustion from trying it the first time around. No gaps were found in the revised proof, and it was published in Annals of Mathematics in 1995, with the title "Modular elliptic curves and Fermat's Last Theorem". As well as the attention of the global media, Wiles received many awards. They include the Rolf Schock Prize, the Ostrowski Prize, the Wolf Prize, the Royal Medal of the Royal Society, the US National Academy of Science's Award in Mathematics, and the Shaw Prize. The International Mathematical Union presented him with a silver plaque, the only time they have ever done so. He was awarded the inaugural Clay

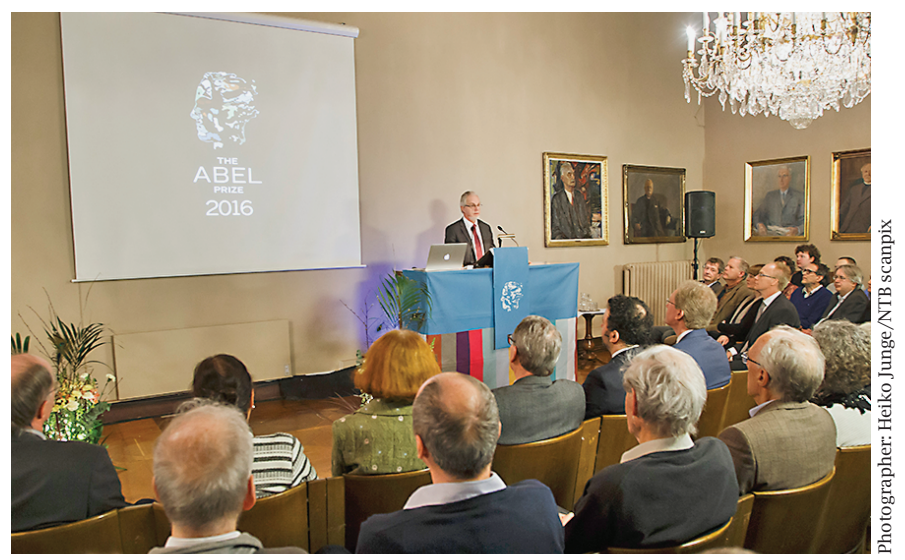

The President of the Norwegian Academy of Science and Letters announces the winner of the 2016 Abel Prize.

Research Award. In 2000 he was given a knighthood. He is a Fellow of the Royal Society and a foreign member of the US National Academy of Sciences and of the French Academy of Sciences.

\section{About the Prize}

The Niels Henrik Abel Memorial Fund was established in 2002 to award the Abel Prize for outstanding scientific work in the field of mathematics. The prize is awarded by the Norwegian Academy of Science and Letters, and the choice of Abel Laureate is based on the recommendation of the Abel Committee. The Abel Committee itself consists of five internationally recognized research scientists in the field of mathematics. The Committee is appointed for a period of two years. The 2015/2016 Abel Committee is:

- Luigi Ambrosio

- Rahul Pandharipande

- John Rognes (Chair)

- Marta Sanz-Solé

- Éva Tardos

Previous recipients of the Abel Prize are:

- Jean-Pierre Serre (2003)

- Michael Atiyah and I. M. Singer (2004)

- Peter Lax (2005)

- Lennart Carleson (2006)

- S. R. S. Varadhan (2007)

- John G. Thompson and Jacques Tits (2008)

- Mikhail L. Gromov (2009)

- John Tate (2010)

- John Milnor (2011)

- Endre Szemerédi (2012)

- Pierre Deligne (2013)

- Yakov Sinai (2014)

- John F. Nash Jr. and Louis Nirenberg (2015). 


\section{Wiles in the Notices}

Ever since Wiles announced his proof of Fermat's Last Theorem in 1993, Notices has published a variety of articles about what was one of the most dramatic events in mathematics in the 20th century.

Below is a list of selected pieces that have appeared in Notices:

July/August 1993: Wiles Proves Taniyama's Conjecture; Fermat's Last Theorem Follows, by Kenneth A. Ribet. https://math. berkeley.edu/ ribet/ Articles/notices.pdf

October 1993: Fermat Fest Draws a Crowd, by Allyn Jackson.

October 1994: Another Step Toward Fermat, by Allyn Jackson www . ams . org/notices/199501/rubin . pdf

July 1995: The Proof of Fermat's Last Theorem by R. Taylor and A. Wiles, by Gerd Faltings www. ams . org/ notices/199507/faltings.pdf

July 1996: Wiles Receives NAS Award in Mathematics, by John Coates www.ams.org/ notices/199607/comm-wi les.pdf

January 1997: Review of BBC's Horizon Program, "Fermat's Last Theorem," reviewed by Andrew Granville www. ams.org/notices/199701/comm-granvi11e. pdf

March 1997: Announcement: 1997 Cole Prize www. ams.org/notices/199703/comm-cole.pdf

November 1997: Paul Wolfskehl and the Wolfskehl Prize, by Klaus Barner www.ams.org/notices/199710/barner.pdf

November 1997: Book Review: Fermat's Enigma by Simon Singh, reviewed by Allyn Jackson. www. ams . org/notices/199710/comm-fermat.pdf

December 1999: Research News: A Proof of the Full Shimura-Taniyama-Weil Conjecture Is Announced, by Henri Darmon www.ams.org/notices/199911/ comm-darmon . pdf

December 2001: Theater Review: Fermat's Last Tango, reviewed by Robert Osserman

www. ams.org/notices/200111/rev-osserman.pdf

September 2005: Wiles Receives 2005 Shaw Prize, by Allyn Jackson www.ams.org/notices/200508/ comm-shaw.pdf
American Mathematical Society

Let Your Voice Be Heard!

\section{Do you enjoy what you're reading in the Notices?}

\section{Would you like to see an article on a certain topic? Person? Situation?}

Then we want to hear from you. Use our Notices Online Commentary Feature to post your general comments and suggestions:

\section{www.ams.org/notices}

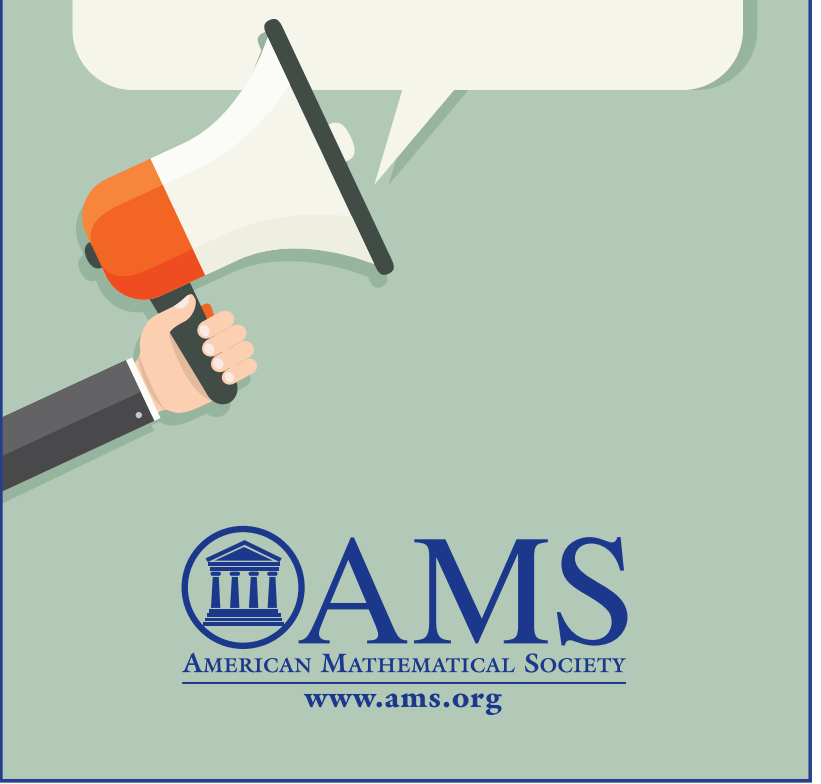

